\title{
Erratum
}

\section{Expression of TGMV antisense RNA in transgenic tobacco inhibits replication of BCTV but not ACMV geminiviruses}

\author{
Eduardo R. Bejarano ${ }^{1}$ and Conrad P. Lichtenstein* \\ Centre for Biotechnology, Department of Biochemistry, Imperial College of Science, Technology \& Medicine \\ Exhibition Road, London SW7 2AZ, UK ( ${ }^{*}$ author for correspondence); 1 present address: Unidad de Genética, \\ Departmento de Biología Celular y Genética, Facultad de Ciencias, Campus Universitario de Teatinos, 29017 \\ Málaga, Spain
}

Plant Molecular Biology 24: 241-248, 1994.

Figure 1 of the above-mentioned paper should have been as follows:

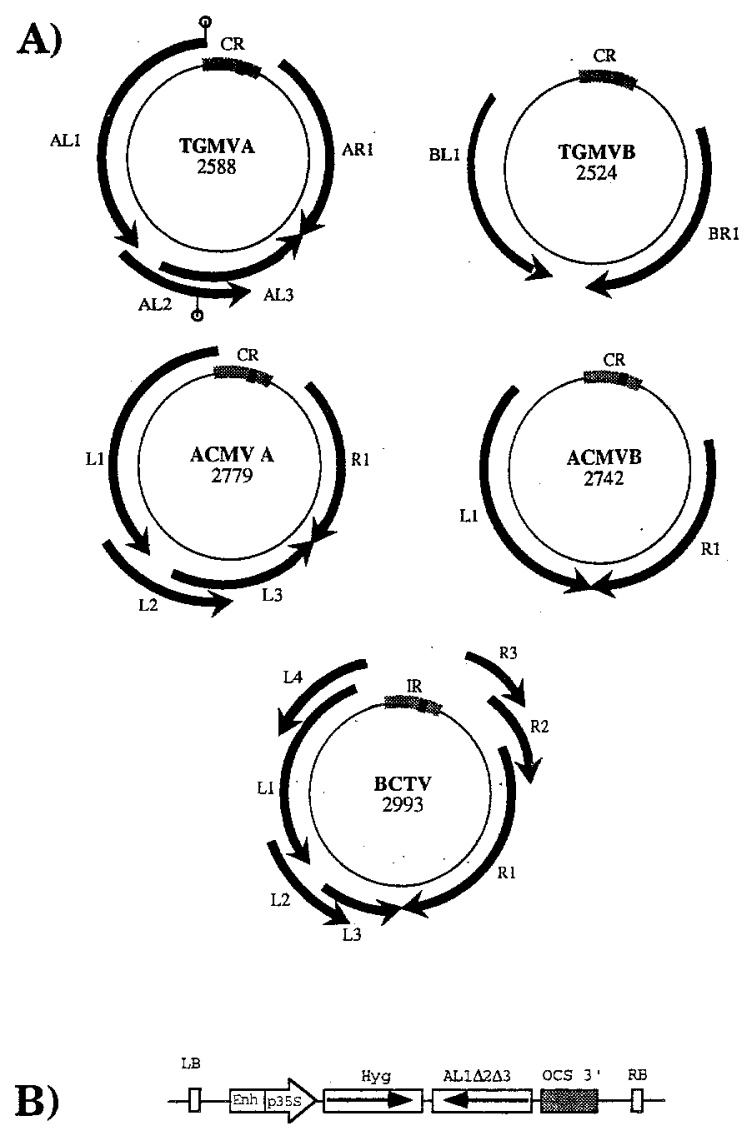

Fig 1. A. Genetic maps of the geminiviruses TGMV [1, 11], ACMV [22] and BCTV [10, 12, 23], with nucleotide lengths of each DNA indicated. A. ca. 200 nucleotide intergenic region (IR), for BCTV, but called the common region (CR) in bipartite viruses (as it is common to both the DNA components) is shaded grey. Within this, a palindromic sequence, yielding a potential stemloop structure, and marked in black, is highly conserved in these geminiviruses. Arrows indicate ORFs, where R (right) refers to those ORFs encoded directly by infecting virion DNA, and L (left) to those by the complementary strand. In TGMV A, the small circles define the region used for the antisense and sense expression cassettes in transgenic plants, this encompasses the entire ORF of $A L 1$ and incomplete ORFs of $A L 2$ and $A L 3$. B. Genetic map of the chimaeric genetic cassette used for the construction of the transgenic tobacco line P2AEN4. Transcription of both the hyg gene (sense orientation) and the $A L I \Delta 2 \Delta 3$ ( $\Delta=$ incomplete) antisense sequences is driven by one cauliflower mosaic virus (CaMV) 'enhanced' $35 \mathrm{~S}$ promoter. The cassette has the $3^{\prime}$ transcriptional regulatory signals of the octopine synthase gene, ocs. Arrows indicate the sense and antisense orientation of the genetic units and RB and LB the T-DNA border sequences (see [5] for details). 